American Journal of Applied Sciences 3 (1): 1634-1639, 2006

ISSN 1546-9239

(C) 2006 Science Publications

\title{
Human-Robot Interface over the Web Based Intelligent System
}

\author{
${ }^{1}$ Desa Hazry, ${ }^{2}$ Masanori Sugisaka and ${ }^{3}$ Taki Yuji \\ ${ }^{1,3}$ Artificial Life and Robotics Laboratory, Electrical and Electronic Engineering \\ Oita University, 700 Oaza Dannoharu, Oita 870-1192, Japan \\ ${ }^{2}$ Department of Electrical and Electronic Engineering, Oita University \\ 700 Oaza Dannoharu, Oita 870-1192, Japan
}

\begin{abstract}
This research extends the capability for the new technology platform by Remote Data Inspection System (RDIS) server from Furukawa Co., Ltd. enabling the integration of standard Hypertext Markup Language (HTML) programming and RDIS tag programming to create a userfriendly "point-and-click" web-based control mechanism. The integration allows the users to send commands to mobile robot over the Internet. Web-based control enables human to extend his action and intelligence to remote location. Three mechanisms for web-based controls are developed: manually remote control, continuous operation event and autonomous navigation control. In the manually remote control the user is fully responsible for the robot action and the robot do not use any sophisticated algorithms. The continuous operation event is the extension of the basic movement from a manually remote control mechanism. In the autonomous navigation control, the user has more flexibility to tell the robot to carry out specific task. Using this method, mobile robot can be controlled via the web, from any places connected to the network without constructing specific infrastructures for communication.
\end{abstract}

Key words: Remote data inspection system (RDIS), user interface, internet robot

\section{INTRODUCTION}

The Internet connects millions of computers all over the world, giving access to communication, data, pictures, videos and even real images of distance environments. However, only a few sample of real physical interaction with distant places through Internet is available at the moment. As the Internet becomes more prevalent in our daily life, many researchers have put their attempts to integrate Internet with robots and build Internet-based robotic systems. The robot operated through the Internet generally uses hardware which includes the robot, the robot work station and other user computers ${ }^{[1]}$. An Internet-controlled robot basically uses the Internet as a command transmission medium and acquiring feedback medium signal ${ }^{[2]}$. The Mercury project ${ }^{[3]}$ is the first Internet robot developed. The TeleGarden system ${ }^{[4]}$ and Mars Pathfinder project $^{[5]}$ were also designed as one of the pioneers in this area. Currently, around the world, a substantial effort is being devoted to developing and controlling robotic devices remotely via the Internet in a wide range of applications. However, current devices are not ready to provide real world services ${ }^{[6]}$. In previous research we improved accuracy of signal extraction from each sensor to build a corresponding independent subsystem $^{[7]}$.

The objective of this research is to develop new technology for user-friendly interface robot control.
The interface can facilitate operators with little background in advance programming language. It can also provide widespread access by only using the "point-and-click" interface available under the standard HTML. The originality is within three windows webbased Internet control mechanisms for the human-robot telecommunication control system. In this way the robot application can be easily operated and the three mechanisms allow the user to realize reliable navigation in the complex environment. Technology RDIS of Furukawa Co., Ltd. and a prototype mobile robot named "Tarou" (Fig. 1) have been merged to demonstrate the efficiency of the proposed user-friendly interface and forming an intelligent control system mobile robot. RDIS is a system capable of relaying information from mobile robot in distance location to a personal computer. This system allows real-time data to be fed into a data base, which makes mobile robot management control system more efficient. This method uses HTML to create the user-friendly interface and inserting RDIS tag programming for mobile robot functionality.

Robot control using Internet basically uses the Internet as command transmission medium as a medium to retrieve feedback signals. The mechanism is remote and generally controlled by a human at the other side.

Remotely operated mechanisms have been used in hazardous environment such as radiation sites ${ }^{[8,9]}$,

Corresponding Author: D. Hazry, Artificial Life and Robotics Laboratory, Electrical and Electronic Engineering, Oita University, 700 Oaza Dannoharu, Oita 870-1192, Japan, Tel: +81-97-554-7831; Fax: +81-97-554-7841 
undersea $^{[10]}$ and space exploration ${ }^{[11]}$. Safaric ${ }^{[12]}$ has made an application of Internet robot for education and training in using expensive equipment. The trainee uses off line virtual environment for task planning which then, exported to remote physical hardware through the internet for robot execution. Recently, teleoperation is being considered for medical diagnosis ${ }^{[13,}{ }^{14]}$, manufacturing ${ }^{[15,16]}$ and micromanipulation ${ }^{[17]}$. Ren et al. ${ }^{[1]}$ gives an excellent review of the issues and opportunities on the teleoperation and telerobotics. Most of these systems were designed for a single specific task and are quite complex. There are also requiring special purpose and highly trained user to control and interact with the mobile robot in the remote environment.

Problems of internet robots: Internet robot systems are facing three major problems ${ }^{[18]}$ :

* The network may cause delays and cannot be guaranteed $^{[19]}$

* The network enables unexperianced users without any background of advance technology to guide the robots

* The web user interface has to be easy to understand and to use in order to attract as many user as possible.

Mobile robot descriptions: Tarou is an intelligent custom autonomous mobile robot with its own control system on-board and has a set of sensors to perceive the environment. The configuration for the mobile robot consists of eight parts, they are briefly as follows; image recognition, sensing to avoid obstacles, PC for control, PC for vision communication, touch panel for display, voice output, motion and power supply. The mobile robot has a differential steering system which comprises two wheels that are individually powered and controlled. Two independently controlled wheels provide both drive and steering. The robot control system is made of three-networked computers: two PCs on board and a notebook (moveable). Two PCs are used for robot control (PC1) and vision processing (PC2). The PCs are connected to the hardware via controller cards and serial ports. The PCs also have Ethernet cards, so that the network message could be used to carry information from one PC to another PC. Two color charge coupled device (CCD) cameras (EVI-G20) mounted on the rotating head. For obstacle detection, a microphone for voice recognition, driving wheel encoders and six ultrasonic sensors are assembled on the mobile robot.

Remote data inspection system (RDIS): The remote control equipment used in mobile robot is a transmitter/receiver RDIS/LT-08 which has eight input ports, eight output ports and RS232 port. Eight output ports of RDIS/LT are connected to eight input ports of the I/O board, IBX-2726C in the computer of robot.

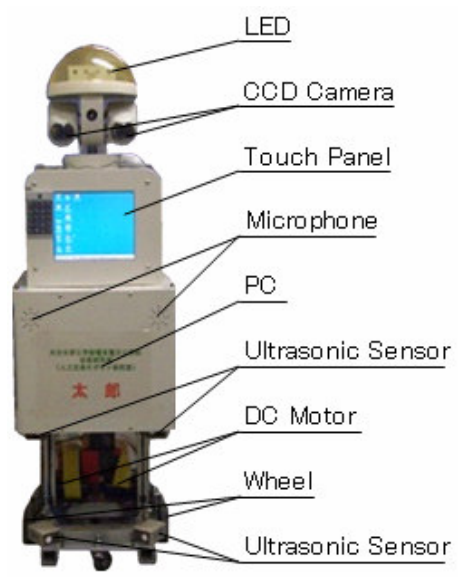

Fig. 1: Mobile robot prototype "Tarou"

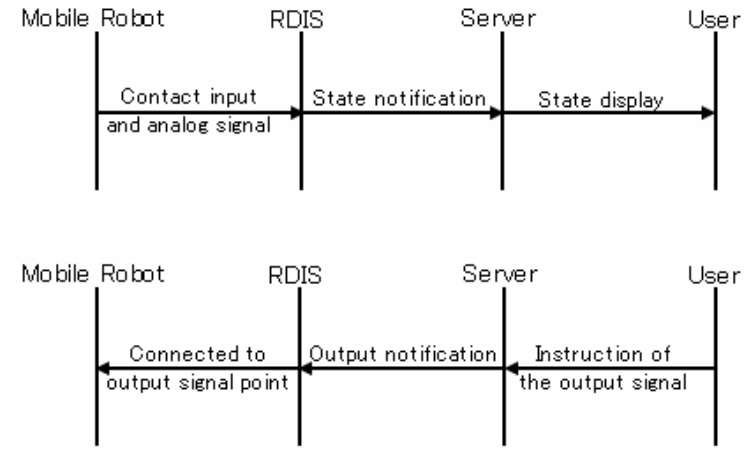

Fig. 2: Outline of the system composition

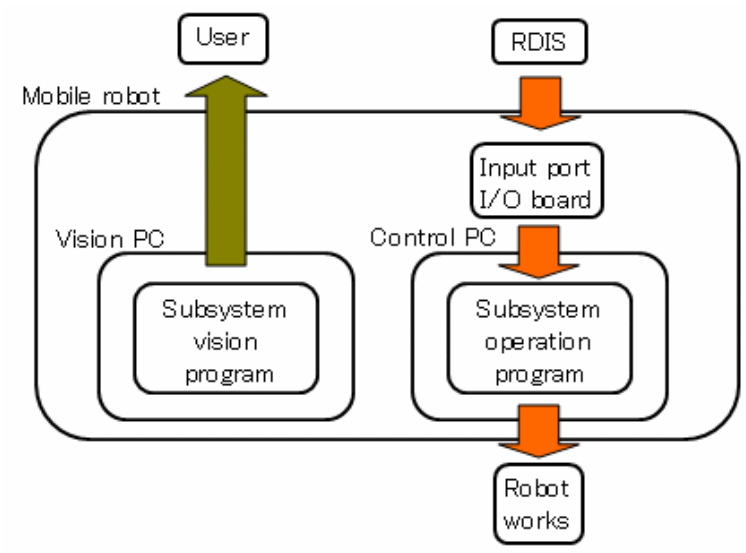

Fig. 3: System architecture

The eight input ports of RDIS/LT are connected to the corresponding ports to check the status of the hardware equipments. Fig. 2 shows the composition of the system. This system carries out its function to enumerate as follows:

* the state of the mobile robot can be observed in real time and

* the output signal can be sent to the mobile robot.

The user sends commands to the mobile robot through the Internet. Upon receipt of the commands, the mobile robot sends feedback on status of commands to the user. Underlying coding tag transmitted by the 
server to robot triggers the Microsoft Visual C++ subsystem program. We developed the subsystem program comprises a) voice control subsystem ${ }^{[20]}$ b) vision subsystem ${ }^{[21,22]}$ c) wheel control subsystem d) head control subsystem and e) camera control subsystem. The connection priority between different subsystems should be parallel and in each system the connection is point to point. Thus, the problem produced by one subsystem will not influence the work of other subsystems ${ }^{[23]}$.

\section{EXPERIMENT}

Web-based control panel user interface: Three windows web-based control mechanisms were developed: 1) manually remote control, 2) continuous operation event and 3) autonomous navigation control. The experiment methodology used here can be summarized by five hierarchical steps.

* The first step determining the user needs and robot tasks.

* Identified the RDIS server tag. (Provided by Furukawa server)

* Prescribed the output signal-the output signal will triggers the event of mobile robot.

* Induce the RDIS server tag into the HTML.

* Upload the completed HTML programming with RDIS server tag to the server and present the HTML in attractive user interface as the user control panel.

Enhancing HTML tag: The function of the system is composed of a port value and a port display. 'Tag Command' is the two character of alphabet command provided by Furukawa server. Such as 'DE' to display the formed button and the button can be "click" to execute the script and ' $\mathrm{IG}$ ' to display the image registered in the server. "Argument" is different according to the each command. Each "Argument" must be put into 「"」(double quotation). $<\mathrm{td}>$ and $</$ td $>$ are part of the HTML programming by means of the RDIS tag is inserted between the standard HTML language. A basic format for enhancing HTML with the RDIS tag is show below:

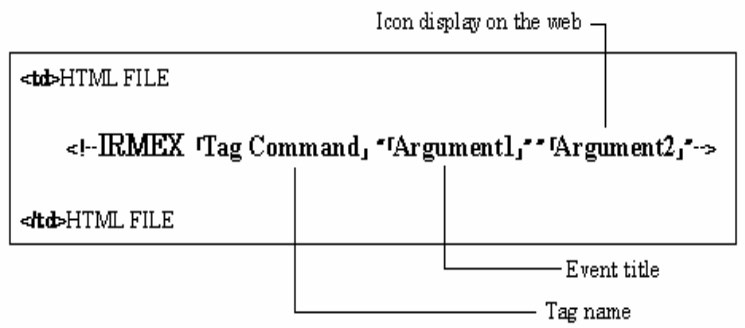

Fig. 4: Basic format of enhancing HTML with the RDIS server tag

In Fig. 4, "Icon display on the web" is use as a click pointer to execute the mobile robot task. Our experiments use the symbol or picture in gif format as a click pointer for easy understanding and attractive to the user. "Event title" (Argument 1) is the task that have been decided for mobile robot to perform action if the user push the icon (Argument 2). The tasks that have been created are shown in Table 1. "Tag name" (tag command) is the command that provided by the Furukawa server.

Number of 16 kinds of movement patterns was created (Table 1) by using output ports 5, 6, 7 and 8 from the RDIS/LT. Each movement pattern is called event. Event title of the output 1 will trigger channel 1 output port of the RDIS/LT. The hexadecimal number is the output value. The output value of 0 gives the signal to stop the movement of 'Tarou' by trigger the wheel control subsystem program in Visual $\mathrm{C}++$ that have been created.

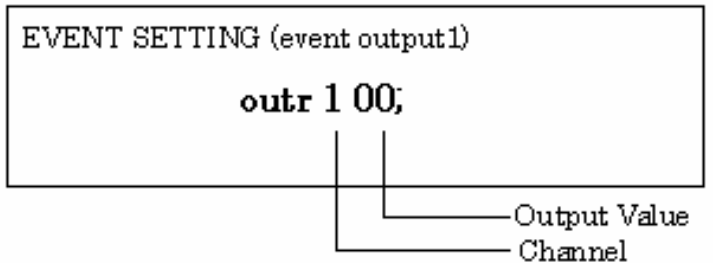

Fig. 5: Channel 1 is correspondence to the out put port number of the RDIS/LT. Output value is the two digits of the hexadecimal number.

\section{RESULTS}

Mechanism 1-manually remote control: In the Mechanism 1, the user is able to send direct commands to the mobile robot such as "Go \#\#\# meter(s)/centimeters(s)", "turn your head left/right", "straighten your head", "look up/down/straight", "turn left/right \#\#\# degrees", "No (for canceling of a command)" (\#\#\# means a number). The user specifies most of the work and he/she has the flexibility to interrupt any commands he/she sends. The tag then must be induced in HTML programming to make mobile robot move with Mechanism 1 as shown below:

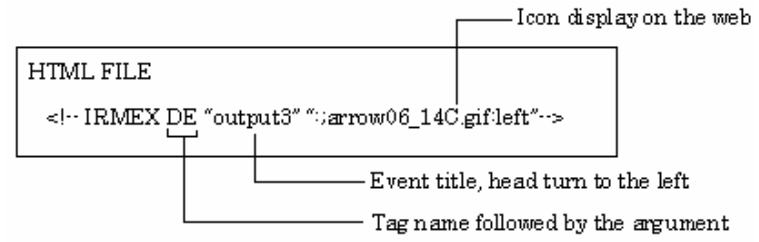

Fig. 6: Manually remote controlled programming structure-Mechanism 1

"output 3" is the Argument 1 where the mobile robot will turn its head to the left (refer to the Table 1). Starting with "1 meter moving forward", "turning head to the right", "turn to the right" and "turning head to the front" with intermittent 6-second waiting intervals in between the each tasks. 
Table 1: RDIS output signal and mobile robot operation event

\begin{tabular}{|c|c|c|c|c|c|c|}
\hline \multicolumn{4}{|c|}{$\begin{array}{l}\begin{array}{l}\text { Output Signal } \\
\text { from RDIS } / \text { LT }\end{array}\end{array}$} & \multirow[t]{2}{*}{ Event Tittle } & \multirow[t]{2}{*}{ Tamu's Movement } & \multirow[t]{2}{*}{ Event Text } \\
\hline 5 & 6 & 7 & 8 & & & \\
\hline 0 & 0 & 0 & 0 & output1 & Stap moving & outr 1 00; \\
\hline a & 0 & 0 & 1 & output? & Initialization & outr 1 10; \\
\hline 0 & 0 & 1 & 0 & output:3 & Turn the head to the left & outr 120 ; \\
\hline a & 0 & 1 & 1 & output4 & Turn the head to the right & outr 1 30; \\
\hline a & 1 & 0 & 0 & output5 & Turn the head to the front & outr 140 ; \\
\hline 0 & 1 & 0 & 1 & outputติ & Turn the COD camera up & outr 150 ; \\
\hline a & 1 & 1 & 0 & output 7 & Turn the GOD camera dawn & outr $160 ;$ \\
\hline a & 1 & 1 & 1 & output: & Turn the $C D Q$ camera forwand & outr 170 ; \\
\hline 1 & 0 & 0 & 0 & outputg & Land mark recognation & outr 180 : \\
\hline 1 & 0 & 0 & 1 & output 10 & Tanou move 1 meter farward & outr $190 ;$ \\
\hline 1 & 0 & 1 & 0 & output 11 & Tarou move 1 meter kackwand & outr $1 \mathrm{a}$; \\
\hline 1 & 0 & 1 & 1 & output12 & Tarou turn left & outr 1 bo; \\
\hline 1 & 1 & 0 & 0 & output 13 & Tarou turn right & outr $1 \mathrm{co}$; \\
\hline 1 & 1 & 0 & 1 & output 14 & Obstacle avoidance & outr $1 \mathrm{dO}$; \\
\hline 1 & 1 & 1 & 0 & output15 & Face tracking and recognitan & outr 1 eo; \\
\hline 1 & 1 & 1 & 1 & Dutput 16 & End the arogramme & outr 1 fo: \\
\hline
\end{tabular}

“:; arrow06_14C.gif:left" is the Argument 2 where referring to the picture of icon in gif format. The icon is displayed out as in HTML for user to use as the push button for controlling the mobile robot. By creating this button the control panel of the user interface would be more attractive and easy to use. In our research we developed 16 basic of movement (Table 1) patterns ${ }^{[24]}$.

Mechanism 2-continuous operation event: In the Mechanism 2, the user executes the task event from the Mechanism 1 continuously. It is necessary to combine the operation of Mechanisms 1 to operate the robot with various pattern of movement. Between each operation a sleep command is inserted. Sleep command is the waiting period of time in between of the each task. The tag induced is shown below:

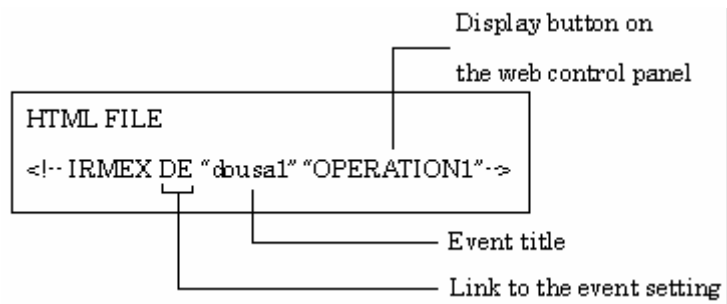

Fig. 7: Continuous operation event programming structure-Mechanism 2

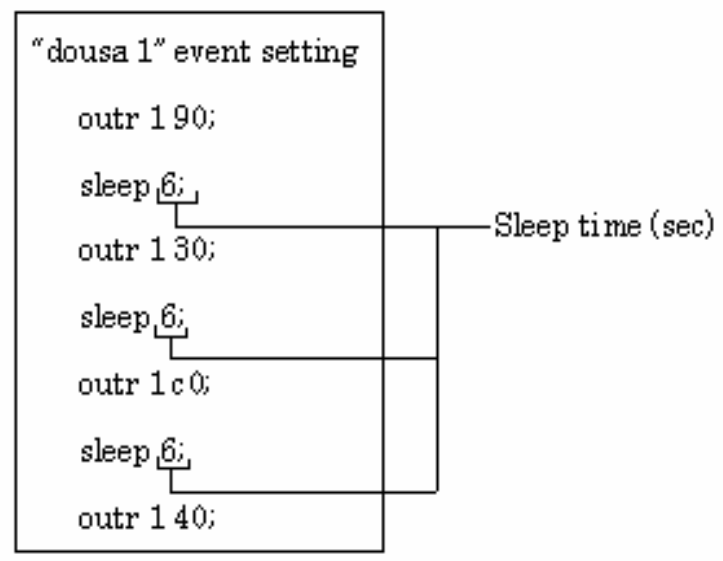

Fig. 8: Event setting inserted by sleep time
By clicking the "OPERATION 1" button the mobile robot will perform a continuous operation

Mechanism 3-autonomous navigation control: In the Mechanism 3, the user only specifies its task and the mobile robot uses its own navigation algorithm to complete a given task. While the robot is moving, the life image taken by the CCD cameras are send back to the user over the internet by wireless LAN connection. In cases where manual control is applied, the robot will not carry out specific operations unless the user gives it step by step commands. This mechanism allows the user to have active and full control of mobile robot, where the user must instruct for the robot to carry out a separate command. In short, the user is full responsibility for the robot actions. The mobile robot does not use any sophisticated algorithms. However, in the autonomous navigation systems Mechanism 3, the user has more flexibility to command the robot to carry out specific tasks. It will use its own navigation algorithm obstacle avoidance. By using this autonomous navigation control method the user can run the obstacle avoidance program from remote distance in Microsoft $\mathrm{VC}++$ that has been written and saved in the operation computer earlier. The tag induced is as follows:

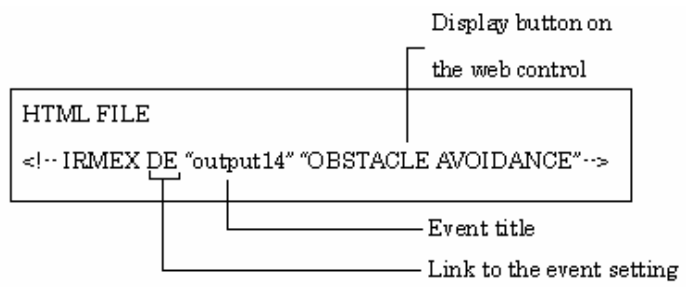

Fig. 9: Autonomous navigation control programming structure-Mechanism 3

For autonomous navigation control mechanism to be reliable and robust, the image taken by CCD camera will be sent to the user immediately. The volume of data coming from the mobile robot and the frequency of the transmission should be synchronized and controlled in order to make the autonomous control work as in the real world without any information delay compare to dynamic movement of the mobile robot.

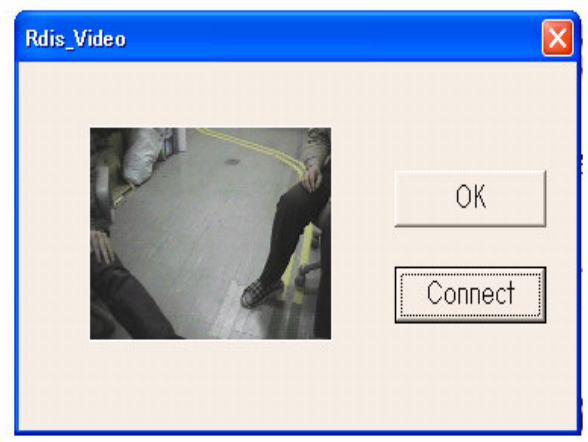

Fig. 10: CCD camera life images display 


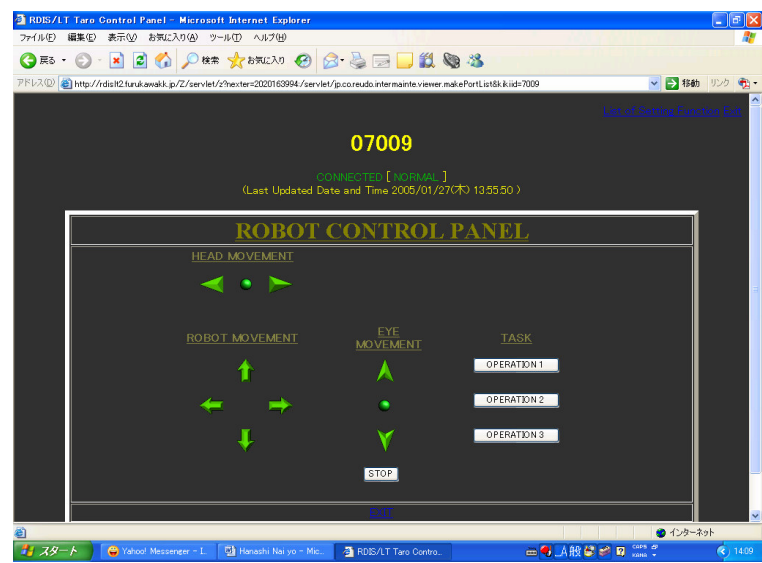

Fig. 11: "Point-and-click" user friendly HTML robot interface control panel

\section{CONCLUSION AND FUTURE WORK}

Mobile robot control consists of advance programming, scientific and high technology. The systematic and methodology aspects of robot control often result in having superficial control design problems that could negatively affect the robot application, usability and appeal. The user-friendly interface of the robot control is extremely advantageous and highly attractive.

Findings of this research reveal that users who are unfamiliar with the advance method can easily operate the mobile robot. With its user-friendly interface, the mobile robot becomes effective and easy to use. Furthermore, the successfully tested three windows web-based Internet control mechanisms were created allow users with no prior programming knowledge to operate the mobile robot. In the experiments, the user successfully guided a mobile robot through a laboratory environment. The user may have full control over the mobile robot with manual control, continuous operation event control and autonomous navigation control. This mechanism was developed to realize reliable navigation in the unknown environment when the robot is unable to deal with the signal directly. Microsoft Visual $\mathrm{C}++$, HTML and RDIS tag are used simultaneously.

However, the current network speed and heavy web traffic pose as teleoperation over the Internet difficulties, which may cause delays in sending live images $^{[2], ~[25-27]}$. Moreover, the computer speed affects the control over the internet very much ${ }^{[28]}$. At this stage, we still have to send the images from CCD Camera to the Internet through wireless LAN card. This does not completely eliminate delays because there are still time delays problems remain in transferring images data through the RDIS and this topic will be addressed in our future work.

\section{REFERENCES}

1. Ren, C.L, L.S. Kuo, H.S. Shen and H.T. Kuo, 2003. Networked intelligent robots through the internet: Issues and opportunities. Proc. IEEE, 91: 371-382.

2. Sugisaka, M. and H. Desa, 2005. Issues and application on robot control using internet. Proc. Intl. Symp. Artificial Life and Robotics, pp: 528531.

3. Goldberg, K. and S. Getner, 2000. The mercury project: A feasibility study for internet robots. IEEE Robotics \& Automation Mag., 7: 35-40.

4. Goldberg, K., M. Mascha, S. Getner, N. Rothenberg, C. Sutter and J. Wiegley, 1995. Desktop Teleoperation via the World Wide Web. Proc. IEEE Intl. Conf. Robotics and Automation, pp: 654-659.

5. Taylor, K. and B. Dalton, 2000. Internet Robots: A new Robotics Niche. IEEE Robotics \& Automation Mag., pp: 27-34.

6. Liu, P.X., M.Q.-H. Meng, J. Gu, S.X. Yang, C. Hu, 2003. Control and data transmission for internet robot. Proc. IEEE Intl. Conf. on Robotics and Automation, pp: 1659-1664.

7. Wang, J. and M. Sugisaka, 2005. Study on the reliable navigation of a mobile robot working under complex environments. Proc. Society of Instrument and Control Engineers, pp: 2901-2903.

9. Cragg, L. and H. Hu, 2003. Application of mobile agents to robust teleoperation of internet robots in nuclear decommissioning. Intl. Conf. IEEE Industrial Technology, 2: 1214-1219.

10. Hong, S.H., J.W. Jeon and J.S. Yoon, 2001. A robot graphic simulator for tele-operation. Proc. IEEE Industrial Electronic, 1: 195-200.

11. Ballard, R.D., 1986. A Last Long Look at Titanic. National Geographic.

12. Hirzinger, G., B. Brunner, J. Dietrich and J. Heindl, 1993. Sensor-based Space RoboticsROTEX and its Telerobotics Features. IEEE Trans. Industrial Electronics, 9: 649-663.

13. Safaric, R., M. Debevc, R.M. Parkin and S. Uran, 2001. Telerobotics Experiments via Internet. IEEE Trans. Industrial Electronics, 48: 424-431.

14. Abolmnesumi, P., S.E. Salcudean, W.H. Zhu, M.R. Sirouspour and S.P. DiMaio, 2002. Image-Guided of a Robot for Medical Ultrasound. IEEE Trans. on Robotics and Automation, 18: 11-23.

15. Takashi, Y., H. Goto and T. Saito, 2002. Health Care System using Face Robot and Tele-operation via Internet. Intl. Conf. Control, Automation, Robotics and Vision, pp: 1025-1030. 
16. Sunil, V.B. and S.S. Pande, 2004. WebROBOT: Internet Based Robotics Assembly Planning System. Intl. J. Computer in Industry, 54: 191-207.

17. Vermeulen, H., T.I. Niekerk, J, Huang and D. Hattingh, 1999. VRML to monitor and control an industries robot via the internet. AFRICON, IEEE Int. Conf., pp: 561-564.

18. Sato, T., J. Ichikawa, M. Mitsuishi and Y. Hatamura, 1994. A new micro-teleoperation system employing a hand-held force feedback pencil. Proc. IEEE Intl. Conf. on Robotics and Automation, 2: 1728-1733.

19. Roland, S. and P. Saucy, 1999. Interacting mobile robots on the web. Workshop Proc. IEEE Intl. Conf. Robotics and Automation.

20. Sheridan, T.B., 1993. Space Teleoperation trough time delay: Review and prognosis. IEEE Trans. Robotics and Automation, 9: 592-606.

21. Kubik, T., M. Sugisaka, 2001. Use of a cellular phone in mobile robot voice control. The Society of Instrument and Control Engineers, pp: 106-111.

22. Wang, J. and M. Sugisaka, 2002. Study on a color based target detection technique. Proc. Intl. Symp. Artificial Life and Robotics, pp: 532-536.
23. Wang, J. and M. Sugisaka, 2002. Study on visualbased indoor navigation for an alife mobile robot. ICSEng., pp: 390-396.

24. Wang, J. and M. Sugisaka, 2005. How to make a mobile robot move more reliably? Proc. Intl. Symp. Artificial Life and Robotics, pp: 4-6.

25. Sugisaka, M. and H. Desa, 2005. User interface in web based communication for internet robot control. Proc. Intl. Conf. Control, Automation and Systems, pp: 49-51.

26. Kikuchi, J., K. Takeo and K. Kosuge, 1998. Teleoperation system via computer network for dynamic environment. Proc. IEEE Intl. Conf. Robotics and Automation, pp: 3534-3539.

27. Huo, X. and J. Su., 2004. New approaches to internet based intelligent robotic system. Proc. IEEE Intl. Conf. Robotics and Automation, pp: 3363-3368.

28. Huo, X. and J. Su., 2004. A distributed architecture for internet robot. Proc. IEEE Intl. Conf. Robotics and Automation, pp: 3357-3362. 